\title{
Correction to: How do Dutch general practitioners detect and diagnose atrial fibrillation? Results of an online case vignette study
}

N. Verbiest-van Gurp ${ }^{1 *}$ (D, D. van Mil ${ }^{1}$, H. A. M. van Kesteren², J. A. Knottnerus ${ }^{1}$ and H. E. J. H. Stoffers ${ }^{1}$

\section{Correction to: BMC Fam Pract (2019) 20:175 \\ https://doi.org/10.1186/s12875-019-1064-y}

Following publication of the original article [1], the authors opted to remove the authors full name from:

N. (Nicole) Verbiest-van Gurp ${ }^{1 *}$, D. (Dominique) van Mil $^{1}$, H.A.M. (Henri) van Kesteren ${ }^{2}$, J.A. (André) Knottnerus ${ }^{1}$, H.E.J.H. (Jelle) Stoffers ${ }^{1}$.

to

N. Verbiest-van Gurp ${ }^{1 *}$, D. van Mil $^{1}$, H.A.M. van Kesteren $^{2}$, J.A. Knottnerus ${ }^{1}$, H.E.J.H. Stoffers ${ }^{1}$.

The original article has been corrected.

\section{Author details}

${ }^{1}$ Care and Public Health Research Institute (CAPHRI), Department of Family Medicine, Maastricht University, Peter Debyeplein 1, 6229 HA Maastricht, Limburg, The Netherlands. ${ }^{2}$ Department of Cardiology, Admiraal de Ruyter Ziekenhuis, s-Gravenpolderseweg 114, 4462 RA Goes, Zeeland, The Netherlands.

Published online: 05 February 2020

\section{Reference}

1. Verbiest-van Gurp N, van Mil D, van Kesteren HAM, et al. How do Dutch general practitioners detect and diagnose atrial fibrillation? Results of an online case vignette study. BMC Fam Pract. 2019;20:175. https://doi.org/10, 1186/s12875-019-1064-y.

The original article can be found online at https://doi.org/10.1186/s12875019-1064-y

* Correspondence: nicole.vangurp@maastrichtuniversity.nl

${ }^{1}$ Care and Public Health Research Institute (CAPHRI), Department of Family Medicine, Maastricht University, Peter Debyeplein 1, 6229 HA Maastricht, Limburg, The Netherlands 